\title{
Sleep Well Be Well: Pilot of a Digital Intervention to Improve Child Behavioural Sleep \\ Problems
}

Professor Harriet Hiscock ${ }^{1,2,3}$, Olivia $\mathrm{Ng}^{1}$, Louise Crossley ${ }^{1}$, Jennifer Chow ${ }^{1}$, Vanessa Rausa ${ }^{1}$, Stephen Hearps $^{1}$

Corresponding Author: Professor Harriet Hiscock, Centre for Community Child Health, Murdoch Children’s Research Institute, The Royal Children’s Hospital, 50 Flemington Road, Parkville, Victoria, 3052. Australia.

\section{Author contact details:}

Professor Harriet Hiscock, email: harriet.hiscock@rch.org.au

Address for all authors: Centre for Community Child Health, Murdoch Children’s Research Institute, The Royal Children’s Hospital, 50 Flemington Road, Parkville, Victoria, 3052. Australia.

\author{
Affiliations \\ ${ }^{1}$ Murdoch Children’s Research Institute, Melbourne, Victoria, Australia \\ ${ }^{2}$ Health Services Research Unit, The Royal Children’s Hospital, Melbourne, Australia \\ ${ }^{3}$ Department of Pediatrics, The University of Melbourne
}

Funding Details: This work was supported by the Royal Children's Hospital Foundation. HH is supported by a National Health and Medical Research Council (NHMRC) Practitioner Fellowship (1136222).

Disclosure Statement: No potential conflict of interest was reported by the authors

This is the author manuscript accepted for publication and has undergone full peer review but has not been through the copyediting, typesetting, pagination and proofreading process, which may lead to differences between this version and the Version of Record. Please cite this article as doi: $10.1111 /$ jpc.15106

This article is protected by copyright. All rights reserved. 


\begin{abstract}
Aims: To investigate whether a digital sleep intervention improves child and caregiver sleep and psychosocial outcomes.

Participants: 120 families with children aged 2-13 years, reporting moderate to severe child behavioural sleep problems, were recruited from a hospital sleep clinic waitlist or the community. Children from non-English speaking families, with known intellectual disability (IQ<70) or severe medical problems excluded.
\end{abstract}

Methods: Tailored behavioural sleep strategies were delivered to primary caregivers via a smart phone app and complementary website. Eligible families completed a baseline questionnaire and child 'sleep check' then received the digital sleep intervention for five weeks, and then completed a post questionnaire. Outcomes: caregiver report of child sleep as no/mild versus moderate/severe problem over past month (primary outcome); problem child sleep patterns (Brief Infant Sleep Questionnaire or Child Sleep Habits Questionnaire), child temperament, caregiver mental health (Kessler 6), caregiver sleep, health service use for their child's sleep, and time off work/activities to access services.

Results: At follow up, caregivers reported fewer moderate/severe child sleep problems (84.6\% to 40.7\%), improved problem child sleep patterns, better temperament and improved caregiver mental health. Caregiver sleep quality and quantity remained unchanged. Health service use (averaged over a six-month period pre and post intervention) fell from $18.9 \%$ pre to $14.1 \%$ post intervention.

This article is protected by copyright. All rights reserved. 
Conclusion: A digital sleep intervention appears promising in improving sleep in children with moderate/severe behavioural sleep problems, and caregiver mental health. It may be a useful alternative to face-to-face management of behavioural sleep problems.

Keywords: Behavioural Sleep Problems; Digital Intervention; Child; Sleep Intervention; Mobile App.

This article is protected by copyright. All rights reserved. 


\section{Introduction}

Sleep problems are common in Australian children affecting up to $30 \%$ of infants ${ }^{1}$ and $40 \%$ of children during the early school years. ${ }^{2,3}$ Most problems are behavioural ${ }^{2,4}$ and include difficulties falling asleep, bedtime resistance, excessive daytime sleepiness and nocturnal wakenings. ${ }^{4,5}$

Persistent sleep problems in children have adverse consequences, including poorer child emotional and behavioural functioning, attention and emotional regulation, ${ }^{1,3}$ parent perception of a 'difficult' temperament, ${ }^{6}$ and poorer parental mental health and health-related quality of life. ${ }^{1,2,3,4}$ Longitudinal studies demonstrate that insufficient sleep or sleep that is of poor quality negatively impacts a child's classroom learning motivation and school function. ${ }^{4}$ Sleep problems are also costly, costing an estimated \$A15.3 million (equivalent to approximately \$A17.3 million in 2020) in additional health service use in Australia every year. ${ }^{7}$

Community-delivered sleep interventions have the potential to save costs to the healthcare system by diverting patients away from tertiary consultations. ${ }^{7}$ Evidence-based behavioural sleep strategies have been shown to improve sleep in typically developing children and those with attention deficit hyperactivity disorder (ADHD). ${ }^{8,9}$ However, face-toface delivery can be costly and have limited availability. ${ }^{10}$ With digital technology advancing and costs plummeting, it has the potential to reduce healthcare costs, increase access and improve outcomes. ${ }^{11}$ 
A systematic review on the effectiveness of digital sleep interventions in adolescents with insomnia found improvements in sleep. ${ }^{12}$ In addition, two trials have shown that internet-based behavioural sleep interventions, delivered to caregivers to help manage their infant's or toddler's behavioural sleep problems, were successful in reducing difficulties with child sleep onset and night wakenings. ${ }^{13,14}$ Mindell et al ${ }^{13}$ also found caregivers who received the digital intervention experienced positive changes in their mental health and sleep. However, none of the trials evaluated a mobile app, none included children in primary school, and none reported on possible flow on benefits to health service use.

We therefore aimed to investigate whether a digital sleep intervention, provided to primary caregivers via a smartphone application and complimentary website, would improve child and caregiver sleep and psychosocial outcomes. We hypothesized that the use of such an app and website would: (1) reduce caregiver report of their child's sleep as a moderate/severe problem; (2) improve caregiver report of their child's problem sleep patterns, child temperament, and caregiver mental health and sleep quality and quantity; and (3) reduce healthcare and family costs.

\section{Methods}

\section{Study design}

This study was a pre-post trial, approved by the RCH Human Research Ethics Committee (HREC 38060G) and registered with the Australian New Zealand Clinical Trials Registry (ACTRN12618001446257).

\section{Eligibility and recruitment}

This article is protected by copyright. All rights reserved. 
Recruitment took place at the RCH Melbourne, Australia, between October 2018 and August 2019. Potential participants were identified via the RCH Sleep Clinic waitlist and via community-based recruitment through the Raising Children Network (government parenting website) parent newsletter and Facebook pages. Interested families provided their contact details via a Registration of Interest form weblink in the advertisements.

To be eligible for the study, children aged 2 to 13 years had to have caregiver reported moderate to severe sleep problems and at least one behavioural sleep disorder, for example, sleep onset association disorder, limit setting disorder, delayed sleep phase, idiopathic insomnia or anxiety leading to insomnia, as identified by initial telephone recruitment screening. Children from non-English speaking families, with a serious medical condition (e.g. severe cerebral palsy), known IQ less than 70 or nonverbal, or suspected obstructive sleep apnea (OSA) were excluded.

The research team telephoned families to introduce the study and further assess eligibility. OSA was assessed by three items from the Child Sleep Habits Questionnaire (CSHQ): “Child snores at night," "Child snorts and/or gasps during sleep,” and "Child seems to stop breathing during sleep.” Families who reported that their child either sometimes (two to four times in a week) or usually (five or more times in a week) snorts and/or gasps during sleep, or sometimes or usually stops breathing during sleep were excluded. ${ }^{3}$ Families who were interested and eligible were then emailed a link to the study information statement, online consent form and baseline questionnaire.

\section{Intervention - Sleep Well Be Well App and Website}

This article is protected by copyright. All rights reserved. 
Upon completion of the online consent form and baseline questionnaire, participants were emailed instructions on how to access the Sleep Well Be Well (SWBW) app and SWBW website guide.

Sleep Well Be Well App

The SWBW app targets common behavioural sleep problems by employing evidencebased strategies ${ }^{15}$ that are standard clinical practice (see supplementary Table 1).

The SWBW app has two main components: the 'sleep check', and the intervention and monitoring phase. The ‘sleep check' comprised a series of questions about the child's sleep problems and behaviours (see Supplementary Table 2). Based on the caregiver's responses, the 'sleep check' recommends a series of strategies tailored to the child's sleep problem(s). In the intervention and monitoring phase, caregivers select which sleep problem(s) they would like to address and are led through the tailored strategies step-by-step (see Figure 2 Bedtime fading strategy snapshot). Families were expected to choose at least one strategy. Strategies are tailored according to the child's age e.g. rewards for preschool and early primary school children are stickers whilst for older children, are raffle tickets swapped for small cash amounts. In general, the app advised families to continue with a strategy for 7-14 days (provided their child was well), before starting a new strategy. Written content with infographics is provided with a daily check in for parents to monitor progress and use of the sleep strategies in the app. The app was co-designed by the lead author in 2018 and Curve Tomorrow - a digital health company co-located with the Murdoch Children's Research Institute 
Sleep Well Be Well Website

The SWBW website provides the same strategies as the SWBW app. However, it does not include a 'sleep check' so information is not tailored to the child's problem(s). Caregivers can read information online or download each strategy as a PDF. The website was hidden from search engines and only accessible to the families via a link provided by the research team.

\section{Measures}

\section{Baseline}

Table 1 outlines study measures. The baseline questionnaire asked caregivers to report on their child's sleep and behaviour, as well as their own sleep and mental health, together with demographic variables. To ascertain the costs of the intervention from healthcare and societal perspectives, caregivers were asked to report their use of sleep-related health services for their child in the last six months, out-of-pocket costs for these services, sleep medications purchased, and if they had taken any time off paid work or missed activities due to their child's sleep problems.

Post

Five weeks post intervention, all families enrolled in the study were emailed a questionnaire including all but the demographic measures from the baseline questionnaire. The primary outcome was caregiver report of child sleep as a moderate/severe problem. ${ }^{9} \mathrm{We}$

This article is protected by copyright. All rights reserved. 
used caregiver report because (1) caregiver perception of a sleep problem is a key driver of help-seeking behaviour and is associated with poorer child mental health and health-related quality of life, (2) the definition of what constitutes a sleep problem from a research perspective is highly debated, (3) subjective measures are more cost-effective and feasible to use than objective measures for determination of child sleep problems in population-based settings, and (4) caregiver report captures behavioural bedtime problems better than actigraphy. ${ }^{5,16}$ Caregivers were also asked to provide feedback on using the SWBW app and website (see Table 1.)

App and website analytics

We used Google analytics to extract data on caregiver use of the website (including number and types of pages accessed), while data on caregiver use of the app including proportion completing the 'sleep check', and the number and type of app strategies accessed was extracted from the database and manually examined by the research team.

\section{Sample size}

A sample size of 91 children, with $80 \%$ power and .05 error level, was sufficiently powered to find an absolute difference of $21 \%$ in the proportion of parents who report their child's sleep as a moderate/severe problem post-intervention.

\section{Data analysis}

Summary statistics described the whole sample, as well as those lost to follow up. Means and standard deviations were used to describe continuous data, with percentages used

This article is protected by copyright. All rights reserved. 
to describe categorical data. For analyses of pre- and post-test differences, Wilcoxon signedrank tests were used for continuous data, and McNemar's test for dichotomous data.

\section{Results}

Of the 197 eligible families, 120 enrolled and two actively withdrew post-enrollment and before sending questionnaires. Just over half of the participating children were male (56\%) and first born (67\%) while most primary caregivers were mothers (96\%) with a university education (70\%) (Table 2).

In terms of engagement, 95 families accessed at least one strategy from the app and 13 families accessed the website only. Only four families did not engage in the intervention at all (see Supplementary Table 3).

\section{Child sleep}

Post intervention, 91 families completed the primary outcome and 74 families completed all outcome measures. Seventy-seven (84.6\%) parents reported a moderate/severe child sleep problem at baseline, which reduced to $37(40.7 \%)$ at follow up ( $<<.001)$. Not all families completed the baseline questionnaire immediately after eligibility screening. This likely reduced the percentage of caregivers reporting their child's sleep problem as moderate or severe at baseline, potentially reducing the effect of the intervention on the outcome.

Mean scores on the subscales of the BISQ significantly improved for average number of night wakings, nocturnal sleep duration and total sleep duration post intervention, but the number of children above clinical cut-off scores for total sleep duration $(\mathrm{n}=2)$ and nocturnal wakefulness $(n=4)$ remained unchanged between time points. A statistically significant 
decrease was observed in the total mean score for the CSHQ from pre (M=55.9, SD=7.6) to post intervention $(\mathrm{M}=48.7, \mathrm{SD}=8.7 ; \mathrm{p}<.001)$, as well as all mean scores for the CSHQ subscales post intervention, except for the Sleep Disordered Breathing subscale. The number of children scoring above the clinical cut point fell from 48 pre to 39 post intervention (Table $3)$.

\section{Child temperament}

Caregiver ratings of their child’s temperament compared to other children, pre and post intervention, are shown in Figure 1. The percentage of caregivers rating their child as “more difficult than average" decreased from 51\% to 36\% post intervention, while the remaining rating options were relatively unchanged.

\section{Caregiver mental health, sleep quality and quantity}

There was a statistically significant decrease in mean caregiver total scores on the K6 from pre $(\mathrm{M}=11.1, \mathrm{SD}=3.9)$ to post digital intervention $(\mathrm{M}=10.3, \mathrm{SD}=3.7 ; \mathrm{p}=.004)$, indicating improved mental health. There were no statistically significant differences in caregiver sleep quality or quantity.

\section{Health service use and costs}

Health service use and costs associated with child sleep problems pre and post digital intervention are shown in Table 4. As our follow-up questionnaire was administered five weeks after families were enrolled, we converted the five-week health service use and costs figures to the equivalent value in a six-month period, assuming linear care. Compared to pre- 
intervention, post intervention, fewer families had sought help for or taken time off work for their child's sleep problems.

\section{Caregiver feedback on app and website use.}

Sixty-eight caregivers completed feedback on the app (see Table 5). More than half reported the app was easy to use, felt confident using the app, did not require face-to-face contact to explain the strategies and were satisfied with the strategies provided for their child's sleep problems. Forty percent of caregivers reported they felt using the app helped their child's sleep problems, although more than half of the caregivers would like to use the app in the future. Most caregivers would recommend the app to other caregivers (81\%).

Fifteen caregivers completed feedback on the website, of which 14 reported they also used the app. Again, the majority reported the website was easy to use and felt confident using the website for their child's sleep problems. Approximately three-quarters of the caregivers reported using the website helped their child's sleep problems (73\%) and most would recommend it to other caregivers (80\%).

\section{Discussion}

A digital sleep intervention, designed to provide caregivers with tailored, evidencebased strategies to manage their child's behavioural sleep problems, was associated with a reduction in caregiver report of child sleep as a problem, problematic child sleep patterns, perception of child temperament as 'difficult' and caregiver mental health symptoms. Most caregivers accessed at least one strategy from the app and website and would recommend the intervention to others. Post intervention, fewer caregivers reported accessing sleep-related 
health services and fewer took time off paid work due to their child's sleep problems. Whilst parents reported a significant improvement in their child’s sleep post app, only $40 \%$ stated that the app helped with their child's sleep problems. It may be that use of the website or supports external to the app also helped caregivers with their child’s sleep.

Our study findings are consistent with research addressing young children’s sleep problems through web-based parenting strategies ${ }^{13,14}$ with reported improvements in child sleep onset, night wakenings, and caregiver mental health. To the best of our knowledge, there are no studies of pediatric digital sleep interventions reporting on outcomes of healthcare and societal costs.

In terms of digital interventions, mobile apps are often utilized to supplement online interventions. ${ }^{17}$ However, research on digital sleep interventions in children has only examined the use of online interventions delivered through a website. Overwhelmingly, caregivers in our study chose to access the sleep intervention via the app and not the website.

Our study had several strengths. It included children aged 2 to 13 years (extending previous digital intervention research restricted to infants and toddlers) with a range of comorbidities, so it is likely results can generalize to most children with behavioural sleep problems. Given the known economic costs of pediatric sleep problems, we extended previous research to report on sleep management costs from healthcare and societal perspectives. We included validated, widely used measures of child and caregiver sleep and psychological outcomes. 
Our study had some limitations. There was no long term follow up, so it is uncertain whether improvements are maintained over time. The study lacked a control group to determine whether the improvements were because of natural change over time. However, in a nationally representative sample of 5107 Australian children followed biennially from ages 0-1 to 10-11 years, using the same measure of moderate/severe child sleep problems, only 9\% of moderate/severe sleep problems resolved in the infant/preschool period suggesting that the natural history of most sleep problems is to persist. ${ }^{18}$ Furthermore, due to variations in how the families engaged in the intervention, it is unclear which components of the intervention resulted in the reported improvements. Finally, a reasonably high attrition rate was observed. Caregivers who withdrew were less well educated than those who did not so results may not generalize to this group. High attrition rates are common in digital health interventions, particularly when the interventions do not involve face-to-face contact. ${ }^{14,17}$

\section{Future research}

Future research should determine whether the initial short-term benefits of this intervention are sustained, clarify which components of the intervention are most successful in improving sleep problems, whether outcomes differ for children with pre-existing psychological disorders, and measure healthcare costs over a comparable period pre and post intervention. Evaluating the app against a control group is desirable but challenging, given the difficulty in preventing control group caregivers from accessing the freely available app. Future studies should also aim to identify those families who benefit from a fully selfdirected app-based intervention from those who require additional assistance. Translating the app would improve equity of access and require additional funding and testing. 


\section{Conclusion and clinical implications}

A digital sleep intervention incorporating tailored, evidence-based strategies, improves caregiver report of sleep and temperament in children with moderate-to-severe behavioural sleep problems as well as caregiver's mental health. The positive effects of this digital intervention may provide a readily accessible intervention for the many children with behavioural sleep problems.

This article is protected by copyright. All rights reserved. 


\section{References}

1. Martin J, Hiscock H, Hardy P, Davey B, Wake M. Adverse associations of infant and child sleep problems and parent health: an Australian population study. Pediatrics. 2007;119(5):947-55.

2. Hiscock H, Canterford L, Ukoumunne OC. Adverse associations of sleep problems in Australian preschoolers: national population study. Pediatrics. 2007;119(1):86-93.

3. Owens JA, Spirito A, McGuinn M, Nobile C. Sleep habits and sleep disturbance in elementary school-aged children. Journal of Developmental \& Behavioral Pediatrics. 2000;21(1):27-36.

4. Quach J, Hiscock H, Canterford L, Wake M. Outcomes of child sleep problems over the school-transition period: Australian population longitudinal study. Pediatrics. 2009;123(5):1287-92.

5. Mindell JA, Kuhn B, Lewin DS, Meltzer LJ, Sadeh A. American Academy of Sleep M. Behavioral treatment of bedtime problems and night wakings in infants and young children. Sleep. 2006;29(10):1263-76.

6. Belmon LS, van Stralen MM, Busch V, Harmsen IA, Chinapaw MJ. What are the determinants of children's sleep behavior? A systematic review of longitudinal studies. Sleep medicine reviews. 2019;43:60-70.

7. Quach J, Gold L, Hiscock H, Mensah F, Lucas N, Nicholson J, et al. Primary healthcare costs associated with sleep problems up to age 7 years: Australian population-based study. BMJ Open. 2013;3(5):e002419.

This article is protected by copyright. All rights reserved. 
8. Hiscock H, Sciberras E, Mensah F, Gerner B, Efron D, Khano S, et al. Impact of a behavioural sleep intervention on symptoms and sleep in children with attention deficit hyperactivity disorder, and parental mental health: randomised controlled trial. BMJ. 2015;350:h68.

9. Quach J, Hiscock H, Ukoumunne OC, Wake M. A brief sleep intervention improves outcomes in the school entry year: a randomized controlled trial. Pediatrics. 2011;128(4):692-701.

10. Manber R, Carney C, Edinger J, Epstein D, Friedman L, Haynes PL, et al. Dissemination of CBTI to the non-sleep specialist: protocol development and training issues. Journal of Clinical Sleep Medicine. 2012;8(02):209-18.

11. Assembly NC. Technology Enabled Care Services: Resource for Commissioners. 2015. 2015.

12. Werner-Seidler A, Johnston L, Christensen H. Digitally-delivered cognitivebehavioural therapy for youth insomnia: A systematic review. Internet Interv. 2018;11:71-8.

13. Mindell JA, Du Mond CE, Sadeh A, Telofski LS, Kulkarni N, Gunn E. Efficacy of an internet-based intervention for infant and toddler sleep disturbances. Sleep. 2011;34(4):451-8.

14. Schlarb AA, Brandhorst I. Mini-KiSS Online: an Internet-based intervention program for parents of young children with sleep problems - influence on parental behavior and children's sleep. Nat Sci Sleep. 2012;4:41-52.

This article is protected by copyright. All rights reserved. 
15. Quach J, Gold L, Arnup S, Sia K-L, Wake M, Hiscock H. Sleep well—be well study: improving school transition by improving child sleep: a translational randomised trial. BMJ Open. 2013;3(10):e004009.

16. Iwasaki M, Iwata S, Iemura A, Yamashita N, Tomino Y, Anme T, et al. Utility of subjective sleep assessment tools for healthy preschool children: a comparative study between sleep logs, questionnaires, and actigraphy. J Epidemiol. 2010;20(2):143-9.

17. Hollis C, Falconer CJ, Martin JL, Whittington C, Stockton S, Glazebrook C, et al. Annual Research Review: Digital health interventions for children and young people with mental health problems-a systematic and meta-review. Journal of Child Psychology and Psychiatry. 2017;58(4):474-503.

18. Williamson AA, Mindell JA, Hiscock H, Quach J. Sleep Problem Trajectories and Cumulative Socio-Ecological Risks: Birth to School-Age. The Journal of Pediatrics. 2019;215:229-37. e4.

19. Sadeh A. A brief screening questionnaire for infant sleep problems: validation and findings for an Internet sample. Pediatrics. 2004;113(6):e570-e7.

20. Sanson A, Prior M, Garino E, Oberklaid F, Sewell J. The structure of infant temperament: Factor analysis of the Revised Infant Temperament Questionnaire. Infant Behavior and Development. 1987;10(1):97-104.

21. Furukawa TA, Kessler RC, Slade T, Andrews G. The performance of the K6 and K10 screening scales for psychological distress in the Australian National Survey of Mental Health and Well-Being. Psychol Med. 2003;33(2):357-62.

This article is protected by copyright. All rights reserved. 
22. Buysse DJ, Reynolds CF, Monk TH, Berman SR, Kupfer DJ. The Pittsburgh Sleep Quality Index: a new instrument for psychiatric practice and research. Psychiatry res. 1989;28(2):193-213.

This article is protected by copyright. All rights reserved. 


\title{
Sleep Well Be Well: Pilot of a Digital Intervention to Improve Child Behavioural Sleep Problems
}

Professor Harriet Hiscock ${ }^{1,2,3}$, Olivia $\mathrm{Ng}^{1}$, Louise Crossley ${ }^{1}$, Jennifer Chow ${ }^{1}$, Vanessa Rausa ${ }^{1}$, Stephen Hearps ${ }^{1}$

Corresponding Author: Professor Harriet Hiscock, Centre for Community Child Health, Murdoch Children's Research Institute, The Royal Children’s Hospital, 50 Flemington Road, Parkville, Victoria, 3052. Australia.

\section{Author contact details:}

Professor Harriet Hiscock, email: harriet.hiscock@rch.org.au

Address for all authors: Centre for Community Child Health, Murdoch Children's Research Institute, The Royal Children’s Hospital, 50 Flemington Road, Parkville, Victoria, 3052. Australia.

\author{
Affiliations \\ ${ }^{1}$ Murdoch Children’s Research Institute, Melbourne, Victoria, Australia \\ ${ }^{2}$ Health Services Research Unit, The Royal Children’s Hospital, Melbourne, Australia \\ ${ }^{3}$ Department of Pediatrics, The University of Melbourne
}

Funding Details: This work was supported by the Royal Children's Hospital Foundation. HH is supported by a National Health and Medical Research Council (NHMRC) Practitioner Fellowship (1136222).

Disclosure Statement: No potential conflict of interest was reported by the authors 


\section{University Library}

\section{- M M N E R VA A gateway to Melbourne's research publications}

Minerva Access is the Institutional Repository of The University of Melbourne

Author/s:

Hiscock, H;Ng, O;Crossley, L;Chow, J;Rausa, V;Hearps, S

Title:

Sleep Well Be Well: Pilot of a digital intervention to improve child behavioural sleep problems

Date:

2020-08-08

Citation:

Hiscock, H., Ng, O., Crossley, L., Chow, J., Rausa, V. \& Hearps, S. (2020). Sleep Well Be Well: Pilot of a digital intervention to improve child behavioural sleep problems. JOURNAL OF PAEDIATRICS AND CHILD HEALTH, 57 (1), pp.33-40. https://doi.org/10.1111/jpc.15106.

Persistent Link:

http://hdl.handle.net/11343/276123 